\title{
Estudio del desempeño atencional en niños en aulas con diferentes acondicionamientos lumínicos
}

\section{Study of attentional performance in children in classrooms with different lighting conditions}

\author{
Juan Manuel Monteoliva 1 ConICET - Celina Graciela Korzeniowski2 CONICET - Mirta Susana Ison ${ }^{3 \text { CONICET }}$ \\ Javier Santillán ${ }^{4}$ CONICET - Andrea Elvira Pattini ${ }^{5 \text { घ CONICET }}$ \\ 1.2.5 Consejo Nacional de Investigaciones Científicas y Técnicas (CONICET), ${ }^{3}$ Universidad del Aconcagua, ${ }^{4}$ Instituto de Investigación en Luz, Ambiente y Visión
}

Argentina

Fecha correspondencia:

Recibido: septiembre 23 de 2015.

Aceptado: junio 14 de 2016.

Forma de citar:

Monteoliva, J. M., Korzeniowski

, C. G., Ison, M. S., Santillán, J.,

\& Pattini, A. E. (2016). Estudio

del desempeño atencional en

niños en aulas con diferentes

acondicionamientos lumínicos. Rev.

CES Psicol., 9(2), 68-79.

Open access

(c) Copyright

Licencia creative commons

Etica de publicaciones

Revisión por pares

Gestión por Open Journal System

ISSN: 2011-3080

DOl: http://dx.doi.org/10.21615/

cesp. 9.2 .5

Sobre los autores:

1. Doctor en Medio Ambiente Visual e lluminación Eficiente. Becario

PosDoctoral del Consejo Nacional de Investigaciones Científicas y Técnicas (CONICET).

Comparte

\section{Resumen}

La iluminación es una de las características físicas más críticas en un espacio de aprendizaje. El objetivo del presente trabajo es evaluar mediante dos instancias experimentales el desempeño atencional en una tarea de detección de detalles monocromáticos en aulas bajo diferentes condiciones de iluminación, contemplando las variables de temperatura de color correlacionada (TCC: $6500^{\circ} \mathrm{K}-4000^{\circ} \mathrm{K}$ ) e iluminancia horizontal (Eh: 300 lx - 500 lx). El diseño experimental empleado fue un experimento intra-sujetos y la muestra estuvo compuesta por 34 alumnos entre 8 y 9 años. Se administró el Test CARAS-R para evaluar eficacia (EA), eficiencia (FA) y rendimiento atencional (RA). Los resultados evidencian diferencias en la $F A(Z=-2.470 ; p=.013)$ y RA ( $Z=-2.385 ; p=0.16)$ de los niños, según las condiciones de TCC. No se registraron diferencias en atención en función de Eh. Promover diseños de acondicionamientos lumínicos eficientes en las aulas es un modo de favorecer el desempeño cognitivo de sus ocupantes.

Palabras claves: Desempeño Atencional, Iluminación, Escuela, Niños.

\section{Abstract}

Lighting is one of the most critical physical characteristics in a learning space. This paper aims to evaluate by two experimental instances attentional performance in a detection task related to monochrome details in classrooms under different lighting conditions, considering correlated color temperature variables (CCT: $6500^{\circ} \mathrm{K}-4000^{\circ} \mathrm{K}$ ) and horizontal illuminance (Ehor: $300 \mathrm{~lx}-500 \mathrm{~lx}$ ). The experimental design was a within-subject design experiment and the sample consisted of 34 students between 8 and 9 years old. CARAS-R Test was administered to assess efficacy (EA), efficiency (FA) and attentional performance. In attentional efficiency $(Z=2470$, $p=.013)$ and attentional performance $(Z=2385, p=0.16)$ of children, significant differences in the conditions of CCT were observed. No differences in Ehor occurred. It was found that providing efficient lighting designs in the classroom may promote its occupants cognitive performance.

Keywords: Attentional Performance, Lighting, Illumination, School, Children. 
Pág 69

\section{Doctora en Psicología. Becaria PosDoctoral del CONICET. \\ 3. Doctora en Psicología. Miembro de la Carrera del Investigador Científico del CONICET y Profesora de la Cátedra de Psicología del Desarrollo de la Facultad de Psicología de la Universidad del Aconcagua (UDA).}

4. Doctor en Psicobiología, Magister en Percepción Visual, Especialista en Medio Ambiente Visual e lluminación Eficiente, Psicólogo.

5. Doctora en Medio Ambiente Visual e lluminación Eficiente. Miembro de la Carrera del Investigador Científico del Consejo Nacional de Investigaciones Científicas y Técnicas (CONICET).

\section{Introducción}

La importancia de la iluminación natural en el interior de un aula radica en el ambiente visual y su incidencia en el desempeño cognitivo del estudiante (Evans \& Ferguson, 2011; Heschong, 1999; Okura, Heschong, \& Wright, 2000; Wu \& Ng, 2003). Estudios sobre la iluminación en escuelas sugieren que la luz natural mejora la atención (Ison \& Pattini, 2009), el comportamiento (Antrop, Roeyers, \& De Baecke, 2005) y el estado de ánimo (Veitch \& Newsham, 1998; Tonello, 1998). Es decir, la iluminación natural no sólo sigue siendo un factor predominante en el entorno percibido (Wienold \& Christoffersen, 2006; Andersen, 2007), que en ciertas condiciones es preferido a una fuente de iluminación artificial (Galasiu \& Veitch, 2006; Boyce. Hunter, \& Howlett, 2003), sino que produce beneficios comprobables también en la salud (Rea, 2002; Lesesne, Visser, \& White, 2003; Webb, 2006). Estudios previos han informado una estrecha relación entre el desempeño atencional y la iluminación natural en escolares (Ison \& Patttini, 2009; Monteoliva, 2014). Asimismo, ha quedado consistentemente documentado que la capacidad atencional es un factor que predice el desempeño escolar de los niños (Checa \& Rueda, 2011; Ison \& Korzeniowski, 2016). De ahí la relevancia de explorar diferentes indicadores de los acondicionamientos lumínicos en edificios educativos y su impacto en la capacidad atencional de los escolares. El valor de estos estudios radica principalmente en la importancia del aprendizaje en la vida de las personas y en el inadecuado acondicionamiento lumínico actualmente existente en muchas de las organizaciones e instituciones de aprendizaje (Monteoliva, 2014).

La atención es uno de los factores más importantes que influye sobre el aprendizaje, en tanto facilita el almacenamiento y recuperación de los contenidos de memoria, permitiendo su actualización cuando la situación lo requiere (Posner \& Rothbart, 2014). Asimismo, interviene en la selección de la información relevante, en el sostenimiento intencional de la misma, en la alternancia del foco atencional entre dos o más fuentes de información, en la manipulación de representaciones mentales y en la modulación de las respuestas a los diversos estímulos (Betts, Mckay, Maruff, \& Anderson, 2006; Ison, Korzeniowski, Segretin, \& Lipina, 2015; Rosselli \& Ardila, 2003). Sin duda, la atención juega un rol clave en el desempeño escolar y correlaciona positivamente con las competencias de lectura, escritura y habilidades matemáticas en escolares primarios (Checa \& Rueda, 2011; Ison, Greco et al., 2015; Waechter, Besner, \& Stolz, 2011; Welsh, Nix, Blair, Bierman, \& Nelson, 2010). En línea con lo expuesto, la literatura señala que los problemas atencionales en la infancia constituyen un factor de riesgo para el fracaso escolar, en tanto afectan negativamente el desempeño social y académico de los niños (Ison, 2011; Polderman, Boomsma, Bartels, Verhulst. \& Huizink, 2010; Posner, Rothbart, \& Rueda, 2011; Sheese, Rothbart, Posner, White, \& Fraundorf, 2008).

A partir de la problemática expuesta, se propone como objetivo de este trabajo evaluar mediante dos instancias experimentales el desempeño atencional en una tarea de detección de detalles monocromáticos en espacios de aprendizaje (aulas) bajo diferentes condiciones de iluminación, contemplando las variables de temperatura de color correlacionada (TCC) e iluminancia horizontal $\left(E_{h}\right)$. La selección de estas variables se fundamenta en su importancia para la percepción humana (Veitch \& Nesham, 1998; Barkman, Wessolowski, \& Schulte-Markwort, 2012). Los parámetros de evaluación de la condición de iluminación (Boyce, 2004) están basados en las normas nacionales del (Instituto Argentino de Normalización y Certificación y la Asociación Argentina de Luminotecnia (IRAM AADL,1994) e internacionales (UNE EN 12464/1, 2012; Certificación LEED-EQ, 2013, entre otras). De esta manera, se 
Pág 70

La atención es uno de los factores más importantes que influye sobre el aprendizaje, en tanto facilita el almacenamiento y recuperación de los contenidos de memoria, permitiendo su actualización cuando la situación lo requiere (Posner \& Rothbart, 2014). busca iniciar una serie de estudios cuantitativos que permitan aportar nuevos conocimientos, con el objetivo de favorecer el adecuado acondicionamiento lumínico en las instituciones educativas. Asimismo, estos estudios permitirían conocer las posibles fluctuaciones atencionales de los niños, asociadas a diferentes condiciones de iluminación, así como también generar nuevas metodologías para la adecuada evaluación de la relación entre iluminación y atención. Así, sería factible identificar condiciones de iluminación óptimas que favorezcan el desempeño atencional de los niños en el contexto escolar.

\section{Metodología}

Los estudios realizados acerca de la iluminación y el desempeño (o productividad) pueden ser convenientemente clasificados en dos grandes grupos: estudios con tareas reales y estudios abstractos (Boyce et al., 2003). Para este trabajo en particular, la experimentación en campo con tareas reales fue seleccionada como criterio. Los experimentos se desarrollaron en un contexto real de aulas, buscando analizar el desempeño atencional de los alumnos en determinadas condiciones de iluminación en su entorno cotidiano.

\section{Caso de Estudio.}

Los experimentos fueron realizados durante el año 2011 en una institución de gestión pública situada a $3 \mathrm{~km}$ de la capital de la provincia de Mendoza, Argentina (32.52.49 S, 68.52.45 E-853msm-). El criterio de selección se basó en tipologías de las escuelas más representativas del parque edilicio educativo de la provincia. Las principales tipologías en la región corresponden a instituciones de gestión pública denominadas compactas y lineales (Ison \& Patttini, 2009; Monteoliva, 2014).

\section{Diseño Experimental}

El diseño experimental empleado fue un experimento intra-sujetos. Dados los inconvenientes comunes a cualquier análisis de una misma población de forma reiterada, en los experimentos fue controlado el orden de presentación de las condiciones experimentales. Asimismo, de manera complementaria, se dividió la muestra en dos sub-muestras ( $\mathrm{A}$ y B) con la intención de aplicar las condiciones en un orden inverso y aleatorio. Esto permitió medir los efectos reactivos de las distintas condiciones.

\section{Participantes}

34 alumnos (22 niños y 12 niñas), con edades entre 8 y 9 años (Media $=8.75, \mathrm{DE}=$ .74); pertenecientes a dos divisiones "A" (55.88\%) (13 hombres y 6 mujeres) y "B" (44.12\%) (9 hombres y 6 mujeres) del turno de la mañana. Los participantes seleccionados para la muestra fueron alumnos de 3er grado de Nivel Inicial de la institución. La selección de este rango de edad se basa en resultados de investigaciones previas, que han observado que tanto la capacidad selectiva de estímulos relevantes (Mezzacappa, 2004; Ison, 2011; Ison \& Carrada, 2011) como la capacidad de filtrar información irrelevante (Arán Filippetti, 2011; Matute, Sanz, Gumá, Roselli, \& Ardila, 2009; Ison, Korzeniowski et al., 2015) exhiben el mayor cambio entre los 5 y 7 años. A partir de esta edad, se produce una considerable disminución de los cambios en el desarrollo de las habilidades atencionales. El procedimiento y los instrumentos empleados contaron con la aprobación del comité de ética del Instituto de Ciencias Humanas, Sociales y Ambientales, dependiente de CONICET-Argentina.

\section{Configuración de los espacios}

Dentro de la institución educativa fueron seleccionadas las aulas (A3a) y (A3b), de similares características morfológicas ( $8.1 \mathrm{~m} \times 6.5 \mathrm{~m} \times 3.8 \mathrm{~m}$ ) (Figura 1). Éstas pre- 
sentan una arquitectura en bloque con un aventanamiento bilateral de orientación norte-sur y galería exterior al norte. La ubicación y dimensiones de las ventanas son: a) en la fachada norte, dos ventanas superiores de $2.3 \mathrm{~m} \times 0.6 \mathrm{~m} \mathrm{y}$, b) en la fachada sur, tres ventanas a la altura de la vista; una de $2.4 \mathrm{~m} \times 1 \mathrm{~m}$ y dos de $1.2 \mathrm{~m} \times$ $1 \mathrm{~m}$. El mobiliario existente durante la evaluación fue el establecido por la institución para cada aula: trece pupitres dobles con sus sillas, ubicados en tres columnas de 5-3-5 filas, respectivamente. El acondicionamiento deseado fue alcanzado durante gran parte del año, sin embargo, para realizar el estudio fueron seleccionadas las jornadas típicas del equinoccio de primavera (con cielo claro, característico de la región), los días 12, 19 y 27 del mes de octubre del año 2011. Previamente el día 14 del mes de septiembre se había realizado una primera aplicación del test a modo de prueba piloto.
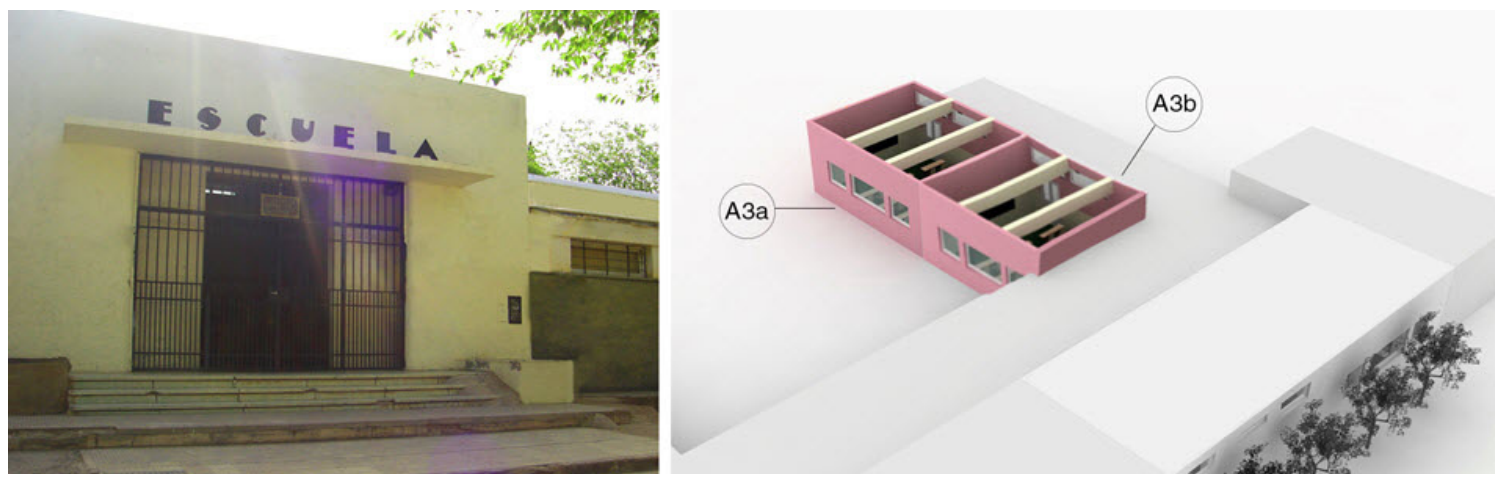

Figura 1. Institución educativa (Mendoza, Argentina). Espacios experimentales seleccionados (A3a y A3b), Ala Norte (N) - orientación (N-S)

Asimismo, son contemplados los parámetros de temperatura de $21^{\circ} \mathrm{C}$ promedio y ruido aproximado de $35 \mathrm{dBA}$; ambos recomendados para la evaluación del desempeño cognitivo (Enander \& Hygge, 1990; Holmberg, Landstrom, \& Kjellberg, 1993). Esta consideración se debe a la correlación encontrada en estudios previos entre la temperatura y el desempeño cognitivo (Knez, 1997). A continuación, se describen las tres condiciones experimentales (Figura 2):

Condición 1: Acondicionamiento lumínico con una temperatura de color correlacionada de $6500{ }^{\circ} \mathrm{K}$ (luz natural) midiéndose una iluminancia horizontal promedio uniforme de 300 lux sobre pupitre.

Condición 2: Acondicionamiento lumínico con una temperatura de color correlacionada de $4000{ }^{\circ} \mathrm{K}$ (luz blanca fría) con una iluminancia horizontal promedio uniforme de 300 lux (valor mínimo por norma) sobre pupitre.

Condición 3: Acondicionamiento lumínico con una temperatura de color correlacionada de $4000^{\circ} \mathrm{K}$ (luz blanca fría) con una iluminancia horizontal promedio uniforme de 500 lux (valor aconsejable por norma) sobre pupitre. 
Condición 1

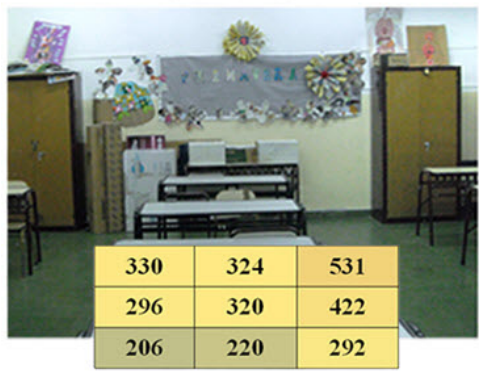

TCC $6500{ }^{\circ} \mathrm{K}$ - Eprom. 300 lux
Condición 2

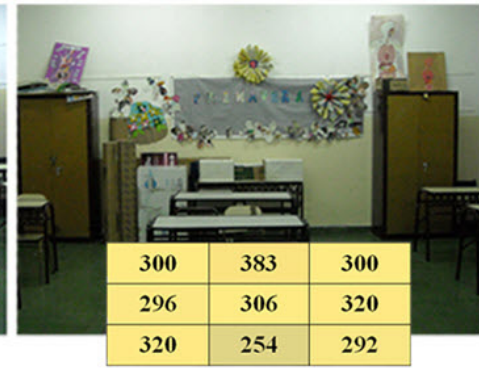

TCC $4000^{\circ} \mathrm{K}$ - Eprom. 300 lux
Condición 3

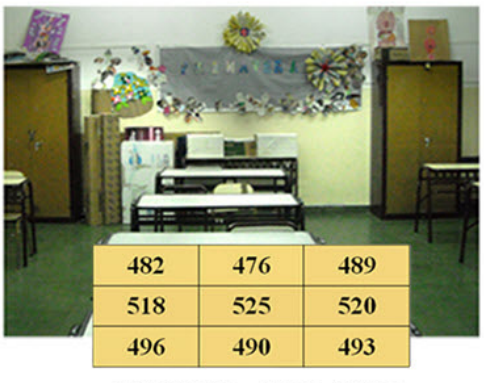

TCC $4000^{\circ} \mathrm{K}$ - Eprom. 500 lux

Figura 2. Condiciones experimentales y variables independientes seleccionadas

\section{Instrumentos de medición}

Se utilizó el Test de Percepción de Semejanzas y Diferencias (TPSD), también denominado "CARAS-R" (Thurston \& Yela, 2012). Este instrumento integra la lista de tests empleados para evaluar la focalización y sostenimiento de la atención (Thurston \& Yela, 2012). Como característica principal, el CARAS-R consta de 60 bloques-estímulos cada uno de ellos formado por tres dibujos esquemáticos de caras (con trazos elementales representando la boca, los ojos, las cejas y el pelo), una de las cuales es distinta. La tarea consiste en determinar cuál es la cara diferente y tacharla. La prueba es aplicable a partir de los 6-7 años en adelante y en todos los niveles socio-económico-culturales. La administración del test CARAS-R es de carácter grupal y contempla tres períodos de tiempo de evaluación: dos tiempos parciales (t1) = 180 seg. y $(\mathrm{t} 2)=\mathrm{T}$-t 1 , y el tiempo total hasta finalizar la tarea $(T)=\mathrm{t} 1+\mathrm{t} 2$. El test ha sido normalizado para escolares mendocinos de 6 a 12 años de edad (Ison \& Carrada, 2011). La consistencia interna del instrumento para la población escolar del Gran Mendoza resultó elevada (rho = 0.87) y fue estimada mediante el procedimiento de partición por mitades a través del coeficiente Spearman-Brown (Ison \& Carrada, 2011).

\section{Parámetros de evaluación}

Los indicadores evaluados corresponden al término de desempeño atencional (Monteoliva, Ison, \& Pattini, 2014). Éste integra los conceptos de Eficacia Atencional (EA) (1) (Ison \& Carrada, 2011), Eficiencia Atencional (FA) (2) y Rendimiento Atencional (RA) (3) (Monteoliva et al., 2014) con el objetivo de profundizar el análisis de las habilidades atencionales en niños, en períodos cortos y prolongados de tiempo, que intervienen en tareas de búsqueda visual y detección de detalles.

$$
E A=\frac{A}{I}=\frac{A}{(A+E)}=\frac{A}{\left(A+E_{o}+E_{c}\right)}
$$

donde (EA) es la eficacia atencional, (A) es el número de aciertos, (I) es el número de intentos y (E) los errores, tanto de omisión (Eo) como de comisión (EC).

(2) $F A=\frac{A}{T}$

donde (FA) es la eficiencia atencional, $(A)$ es el número de aciertos y (T) el tiempo total requerido para realizar la tarea 
(3) $\quad R A=\frac{E A}{T^{0.2}}$

donde (RA) es el rendimiento atencional, (EA) es la eficacia atencional del sujeto en la tarea y $(T)$ el tiempo requerido para realizar la tarea.

\section{Caracterización visual del estímulo.}

a) diseño del estímulo y la tarea sobre soporte impreso, b) tiempos: parciales (t1) y (t2); y tiempo total (T) hasta finalizar la tarea, c) color del optotipo 69.21.8.0 (CMYK), d) distancia aproximada al test 0.3 [m], e) contraste del optotipo 0.45 .

\section{Procedimientos}

Para realizar el estudio se contó con el aval de la Dirección General de Escuelas (DGE) de la provincia de Mendoza y la autorización de los directivos de la escuela participante. Se envió una nota escrita a los padres de los alumnos, en la cual se explicaron los objetivos del estudio y se les solicitó el consentimiento para la participación de sus hijos. Por otra parte, se explicó a los niños autorizados las características de las tareas a realizar, se los invitó a participar voluntariamente y se les informó sus derechos como participantes, obteniéndose su consentimiento.

La administración del test CARAS-R fue de carácter grupal, y estuvo a cargo de psicólogos y profesionales especializados en evaluación cognitiva en niños. Este instrumento cuenta con un período de práctica que le permite al administrador asegurarse que el niño ha comprendido las instrucciones. Luego, se da comienzo a la prueba. Al contener la misma dos periodos (t1) y (t2), se emplea para la discriminación de éstos un cambio de lápiz de color en la ejecución. Un color se utiliza para completar la tarea durante los primeros $180 \mathrm{seg}$. (t1) y el otro, desde los $181 \mathrm{seg}$. hasta finalizar la tarea (t2). Este procedimiento permite conocer los bloques tri-estímulos correspondientes a cada uno de los tiempos parciales estudiados.

Los criterios metodológicos empleados en la administración del instrumento son el resultado del análisis de antecedentes y estudios previos realizados en instituciones educativas (Küller \& Lindsten, 1992; Heschong, Roger, \& Okura, 2002). A partir de ello, las mediciones son realizadas a mediados de semana reduciendo posibles impactos de factores ajenos a los estudiados. El período de toma seleccionado fue de 9:40 a.m a 10:40 a.m. (2do Módulo - Turno Mañana), intervalo requerido para los acondicionamientos por fuente natural, así como también, posterior al recreo y desayuno diario (servido por la escuela). Esta consideración radica en la demostrada influencia del desayuno en el desempeño atencional de los alumnos (Wesnes, Pincock, Richardson, Helm, \& Hails, 2003). Los tiempos de adaptación al entorno también se consideraron para la configuración de los acondicionamientos lumínicos. Las intervenciones a los espacios fueron generadas el día previo a la instancia experimental. Esto permite que, al llegar los alumnos a la institución al día siguiente, ingresen y permanezcan en estos espacios acondicionados aproximadamente una hora y media antes de la aplicación de las pruebas. Esta instancia experimental fue realizada en tres tomas (tres semanas), los días 12, 19 y 27 del mes de octubre del año 2011. Asimismo, los participantes son informados de los objetivos y fines del estudio para "mantener al margen" sus creencias previas acerca de los parámetros

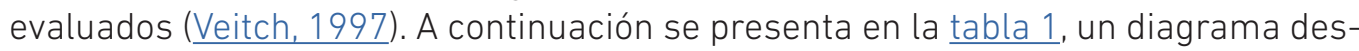
criptivo del orden y aplicación aleatorizada de los acondicionamientos lumínicos y las tomas realizadas. 
Tabla 1. Orden y aplicación aleatorizada de los acondicionamientos lumínicos

\begin{tabular}{|c|c|c|c|c|}
\hline \multirow{2}{*}{ Muestra } & \multirow{2}{*}{$\begin{array}{c}\text { Sub- } \\
\text { muestra }\end{array}$} & \multicolumn{3}{|c|}{ Experimento 1 (2011) } \\
\hline & & 1ra Toma & 2da Toma & 3er Toma \\
\hline \multirow{2}{*}{$\begin{array}{l}\text { 3er } \\
\text { Grado }\end{array}$} & $\begin{array}{l}\text { División A } \\
(n=19)\end{array}$ & $\begin{array}{l}\text { TCC } 6500{ }^{\circ} \mathrm{K} \\
\text { Eh }_{\text {prom }} 300 \text { lux }\end{array}$ & $\begin{array}{l}\text { TCC } 4000^{\circ} \mathrm{K} \\
\text { Eh }_{\text {prom }} 500 \text { lux }\end{array}$ & $\begin{array}{l}\text { TCC } 4000^{\circ} \mathrm{K} \\
\text { Eh }_{\text {prom }} 300 \text { lux }\end{array}$ \\
\hline & $\begin{array}{l}\text { División B } \\
(n=15)\end{array}$ & $\begin{array}{l}\text { TCC } 4000^{\circ} \mathrm{K} \\
\text { Eh }_{\text {prom }} 300 \text { lux }\end{array}$ & $\begin{array}{l}\text { TCC } 6500^{\circ} \mathrm{K} \\
\text { Eh }_{\text {prom }} 300 \text { lux }\end{array}$ & $\begin{array}{l}\text { TCC } 4000^{\circ} \mathrm{K} \\
\text { Eh }_{\text {prom }} 500 \text { lux }\end{array}$ \\
\hline
\end{tabular}

\section{Análisis de datos}

En primera instancia se exploraron los datos mediante estadística descriptiva (media y desviación estándar). Luego, se analizó el cumplimiento de los supuestos de normalidad univariada de las variables a través de los índices de asimetría y curtosis. Debido a que algunas variables no mostraron una distribución semejante a la curva normal, se decidió emplear pruebas no paramétricas para el análisis de los datos. Las pruebas no paramétricas aplicadas fueron: prueba de Friedman para varias muestras relacionadas y análisis post hoc de Wilcoxon. Asimismo, fue aplicado el ajuste de Bonferroni, debido a las comparaciones múltiples realizadas y a la necesidad de controlar el error Tipo I. Este ajuste es calculado a través del nivel de significación propuesto (0.05), dividido en el número de pruebas o condiciones ejecutadas, corrigiendo así el valor de significación a $p<.017$.

\section{Resultados}

Para dar respuesta al objetivo del estudio, se comparó el desempeño atencional de los niños en tres condiciones lumínicas focalizadas en las variables independientes temperatura de color correlacionada (TCC) e iluminancia horizontal $\left(\mathrm{E}_{\mathrm{h}}\right)$. Los resultados obtenidos mediante la prueba de Friedman presentaron diferencias estadísticamente significativas en los indicadores de atención evaluados en las diferentes condiciones experimentales, $\chi^{2}(20)=606.97, p=0.000$. Si bien esta instancia experimental cuenta con tres condiciones, a nivel teórico el estudio está focalizado en dos variables independientes: temperatura de color correlacionada (TCC) e iluminancia horizontal promedio $\left(E_{\text {prom }}\right)$. Por este motivo el análisis de Wilcoxon presenta dos pares de análisis correspondientes a cada una de estas variables (ver Tabla 2).

Tabla 2. Comparación de los indicadores de desempeño atencional en niños, según temperatura de color correlacionada e iluminancia horizontal promedio

\begin{tabular}{|c|c|c|c|c|c|c|c|}
\hline \multirow[b]{2}{*}{ Tpo } & \multirow[b]{2}{*}{ Prueba de Wilcoxon } & \multicolumn{3}{|c|}{ Par 1 (TCC) } & \multicolumn{3}{|c|}{ Par 2 (Eh $\left.{ }_{\text {prom }}\right)$} \\
\hline & & $E A$ & $F A$ & $R A$ & $E A$ & $F A$ & $R A$ \\
\hline \multirow{2}{*}{$\mathrm{t} 1$} & Z & $-1.921^{a}$ & & & $-1.048^{a}$ & & \\
\hline & Asymp. Sig. (2-tailed) & .055 & & & .294 & & \\
\hline \multirow{2}{*}{$\mathrm{t} 2$} & Z & $-1.421^{a}$ & $-2.026^{a}$ & $-2.282^{a}$ & $-1.206^{b}$ & $-.795^{b}$ & $-.333^{b}$ \\
\hline & Asymp. Sig. (2-tailed) & .155 & .043 & .022 & .228 & .427 & .739 \\
\hline \multirow{2}{*}{$\mathrm{T}$} & Z & $-1.665^{a}$ & $-2.470^{a}$ & $-2.385^{a}$ & $-.325^{b}$ & $-.231^{\mathrm{a}}$ & $-.231^{\mathrm{a}}$ \\
\hline & Asymp. Sig. (2-tailed) & .096 & $.013^{*}$ & $.016^{*}$ & .745 & .817 & .817 \\
\hline
\end{tabular}

* valores considerados estadísticamente significativos $p<.017$ 
Pág 75

Estos resultados indican que un acondicionamiento con una TCC de $4000^{\circ} \mathrm{K}$ podría ser uno de los aspectos que favorece la capacidad cognitiva para seleccionar y mantener la atención en tareas de detección de detalles (monocromática) en escolares, lo que implicaría que ellos utilizan los mínimos recursos de tiempo disponibles y con ello mejoran su rendimiento atencional (RA)
Par 1: TCC $6500^{\circ} \mathrm{K}$ (condición 1) vs TCC $4000^{\circ} \mathrm{K}$ (condición 2). Los resultados obtenidos de la prueba de Wilcoxon evidenciaron diferencias estadísticamente significativas en el período total de tiempo $(T)$ en los indicadores de eficiencia atencional $(F A)(Z=-2.470, p=.013)$ y rendimiento atencional $(R A)(Z=-2.385, p=.017)$ con una diferencia de medias en ambos casos de .02 (es decir, $F A: M=0.13 ; M=0.11$ y RA: $M$ $=0.27 ; \mathrm{M}=0.25$, condición 1 y 2 respectivamente) .

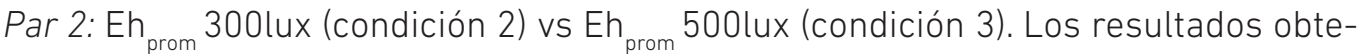
nidos en la prueba de Wilcoxon evidenciaron que el incremento en la iluminancia horizontal sobre pupitre con una fuente artificial de 300 lux a 500 lux - bajo condición de TCC de $4000^{\circ} \mathrm{K}$-, no presentó diferencias estadísticamente significativas en ninguno de los indicadores de desempeño atencional (EA, FA y RA) (ver Tabla 2).

\section{Discusión}

El artículo presenta resultados obtenidos en la evaluación del desempeño atencional en espacios de aprendizaje (aulas) bajo diferentes condiciones de iluminación. Tal como sostiene Boyce (2006), este tipo de abordaje permite abandonar el estudio del uso de la iluminación basado tan sólo en las mejoras de visibilidad y disminución de las molestias visuales e iniciar estudios integrales más "honestos".

En primera instancia fue evaluada la temperatura de color correlacionada (TCC) de $4000{ }^{\circ} \mathrm{K}$ (luz blanca fría) y $6500{ }^{\circ} \mathrm{K}$ (luz natural), ambas empleadas usualmente en los edificios educativos de la región (Mendoza, Argentina). Los resultados obtenidos evidencian diferencias estadísticamente significativas a favor de la TCC de $4000^{\circ} \mathrm{K}$ en los indicadores de eficiencia atencional (FA) y rendimiento atencional (RA) en períodos prolongados de tiempo. En otras palabras, estos resultados indican que un acondicionamiento con una TCC de $4000^{\circ} \mathrm{K}$ podría ser uno de los aspectos que favorece la capacidad cognitiva para seleccionar y mantener la atención en tareas de detección de detalles (monocromática) en escolares, lo que implicaría que ellos utilizan los mínimos recursos de tiempo disponibles y con ello mejoran su rendimiento atencional (RA). Los resultados hallados están en línea con investigaciones anteriores las cuales han informado la importancia de la TCC como un factor esencial en espacios de aprendizaje fomentando y mejorando el impacto de la iluminación en los usuarios (Sanaz, 2011). En el caso de un uso inadecuado de la TCC, ésta presenta efectos negativos sobre la salud humana, tanto a nivel ocular, como emocional y en el sistema circadiano (Kenz, 1997; Van Bommel \& Van Den Beld, 2004; Mills, Tomkins, \& Schlangen, 2007; Halonen, Tetri, \& Bhusal, 2010), que podrían producir una pérdida adicional del desempeño en el aula.

La atención, en tanto sistema de control cognitivo, facilita la activación y el funcionamiento de otros procesos psicológicos para ejecutar eficazmente las tareas, siendo uno de los factores más importantes vinculados al desempeño escolar infantil. Por esta razón, este trabajo se incluye entre los estudios cuantitativos orientados a aportar nuevos conocimientos sobre el adecuado acondicionamiento lumínico en las instituciones educativas como promotor del desempeño escolar.

En segunda instancia fue evaluada la iluminancia horizontal promedio (Eh $\mathrm{prom}$ ) mínima (300lux) y aconsejable (500lux) por la normativa nacional vigente en Edificios Educativos IRAM AADL J20 04. Los resultados obtenidos no evidencian diferencias estadísticamente significativas en el desempeño atencional (EA, FA y RA) de los escolares bajo diferentes condiciones de iluminancia. Este hallazgo es congruente con estudios previos realizados con tareas visuales (Boyce, 2004) donde los incrementos 
Pág 76

Es necesario un cambio de paradigma -como propone Boyce (2006)-, de modo que la iluminación no sólo sea evaluada por su uso, sino también por la relación que ésta establece con la visibilidad y el desempeño de sus ocupantes.

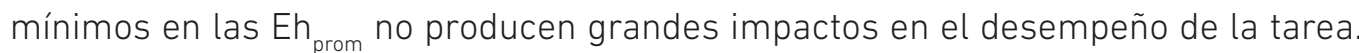
A partir de estos datos se generan nuevos interrogantes: en el caso de utilizar estímulos cromáticos o replicar el experimento en distintos períodos estacionales, ¿las variables lumínicas consideradas brindarían los mismos resultados?

El trabajo experimental que se informa presenta la limitación de ser un estudio piloto sobre una muestra pequeña de 34 alumnos. Sin embargo, marca un precedente en la evaluación de las habilidades atencionales en acondicionamientos dinámicos, característicos de los espacios iluminados principalmente por luz natural. Estos resultados provienen de niños y niñas de escuelas estatales urbanas por lo cual la interpretación de los resultados debe ser contextualizada. Además, aunque puede contarse con un primer indicador, son necesarias evaluaciones complementarias para un adecuado diagnóstico atencional.

A pesar de las limitaciones mencionadas, este estudio brinda continuidad a trabajos anteriores sobre iluminación y su relación con la atención (Ison \& Pattini, 2009), siendo su aporte específico profundizar en diferentes indicadores de iluminación en aulas y su influencia en parámetros específicos del desempeño atencional (EA, FA y RA), evaluados en períodos cortos y prolongados de tiempo. En relación al primer aspecto, los resultados obtenidos en este trabajo en consonancia con normativas internacionales anteriormente mencionadas, buscan incorporar nuevos parámetros en la normativa argentina IRAM AADL J20 04 -como la TCC- en la evaluación de los acondicionamientos lumínicos en espacios de aprendizaje. Asimismo, en relación a los instrumentos de medición de la atención, este trabajo ofrece una serie de indicadores para evaluar el desempeño atencional (Monteoliva et al. 2014) de los niños en tareas de lápiz y papel. En este contexto, estas nuevas expresiones no sólo incorporan la exactitud del niño al momento de realizar la tarea (EA), sino también los tiempos requeridos para ello (FA); integrando ambas capacidades en un concepto (RA). Estos indicadores buscan sensibilizar el instrumento para valorar las fluctuaciones atencionales en niños en diferentes condiciones de iluminación. La integración de estos dos aspectos generó nuevos antecedentes y nuevos interrogantes para el desarrollo de futuros estudios que permitan establecer y normalizar criterios básicos de acondicionamientos lumínicos que favorezcan el desempeño atencional de los niños. A partir de los resultados obtenidos se abren nuevas perspectivas, que no sólo deberán incorporar al estudio nuevas tecnologías de iluminación artificial complementaria (p.e. LED, dimerizado, entre otros), sino también integrar al análisis cuantitativo de las evaluaciones psicológicas, estudios subjetivos de preferencias de los usuarios a estos espacios.

\section{Conclusiones}

Como ha sido desarrollado a lo largo del trabajo, desde diferentes perspectivas (confort, salud, energética) se demuestran los beneficios de lograr un mejor aprovechamiento de la luz natural en las instituciones educativas. Sin embargo, el uso de la luz natural sin control, la iluminación deficiente y la falta de mejoras en las instalaciones lumínicas son problemas permanentes en muchas organizaciones e instituciones de aprendizaje. Asimismo, se conoce que los aportes de la luz natural exceden la "utilidad" de fuente de iluminación para maximizar la visibilidad y minimizar las molestias visuales. Por este motivo, es necesario un cambio de paradigma -como propone Boyce (2006)-, de modo que la iluminación no sólo sea evaluada por su uso, sino también por la relación que ésta establece con la visibilidad y el desempeño de sus ocupantes. En esta línea de estudio, el aporte de este trabajo es haber 
estudiado, en un contexto natural, el impacto de diferentes condiciones de iluminación en el desempeño atencional de los niños. A partir de estos resultados y nuevos estudios en ejecución se busca ayudar a los psicólogos a considerar las condiciones lumínicas del aula como variable importante para la adecuada administración de instrumentos psicométricos y la evaluación de la atención. Por otro lado, se busca ayudar a profesionales proyectistas a fomentar diseños de espacios de aprendizaje con acondicionamientos lumínicos por fuente natural eficiente, que favorezcan el desempeño cognitivo de sus ocupantes.

\section{Referencias}

Andersen, M. (2007). Pioneering Daylighting Design Tool to combine Architectural Form with Advanced Technology: The LightSolve Project. Massachusetts: Building Technology Program. http://www.scielo.br/scielo.php?script=sci arttext\&pid $=$ S1678-86212013000400016

Antrop, I., Roeyers H., \& De Baecke, L. (2005). Effects of Time of Dayon Classroom Behavior in Children with ADHD. School Psychology International, 26(1), 29-34. http:// www.scielo.br/scielo.php?script=sci arttext\&pid=S1678-86212013000400016

Arán Filippetti, V. (2011). Funciones ejecutivas en niños escolarizados: efectos de la edad y del estrato socioeconómico. Avances en Psicología Latinoamericana, 29(1), 98-113. www.scielo.org.co/pdf/apl/v29n1/v29n1a08.pdf

Barkman, C., Wessolowski, N., \& Schulte-Markwort, M. (2012). Applicability and efficacy of variable light in schools. Physiology and Behavior, 105, 621-627. doi: 10.1016/j.physbeh.2011.09.02.

Betts, J., Mckay, J., Maruff, P., \& Anderson, V. (2006). The development of sustained attention in children: The effect of age and task load. Child Neuropsychology, 12, 205-221. Google

Boyce, P. R. (2004). Lighting research for interiors: The beginning of the end or the end of the beginning. Lighting Research \& Technology, 36, 283-294. https://www. researchgate.net/publication/245385276 Lighting research for interiors The beginning_of the_end_or_the end_of the_beginning

Boyce, P. (2006). Light and health: the implications for lighting. Daylight and Architecture Magazine, Velux (autumn), 40-43. http://2012.experiencinglight.nl/ doc/3.pdf

Boyce, P., Hunter, C., \& Howlett, O. (2003). The Benefits of Daylight through Windows. New York: Lighting Research Center. Rensselaer Polytechnic Institute. http:// thedaylightsite.com/wp-content/uploads/papers/DaylightBenefits.pdf

Checa, P., \& Rueda, M. R. (2011). Behavioral and Brain Measures of Executive Attention and School Competence in Late Childhood. Developmental Neuropsychology, 36(8), 1018-1032. doi: 10.1080/87565641.2011.591857

Enander, A. E., \& Hygge, S. (1990). Thermal stress and human performance. Scandinavian Journal of Work Environment and Health, 16, 44-50. www.sjweh.fi/download.php?abstract id $=1823 \&$ file nro $=1$

Evans, G.W., \& Ferguson, K.T. (2011). Built Environment and Mental Health. En Jerome ON, Editor(s)-in-Chief, Encyclopedia of Environmental Health, Elsevier, Burlington, (pp.s 446-449). doi: 10.1016/B978-0-444-52272-6.00006-4.

Galasiu, A. D., \& Veitch J. A. (2006). Occupant preferences and satisfaction with the luminous environment and control systems in daylit offices: a literature review. Energy and Buildings, 38(7), 728-742. https://www.researchgate.net/publication/222580833 Occupant preferences and satisfaction with the luminous environment and control systems in daylit offices A literature review 
Halonen, L., Tetri, E., Bhusal, P. (2010). Chapter 3: Lighting Quality. En Guidebook on energy efficient electric lighting for buildings, (pp. 41-56). Finland: Aalto University School of Science and Technology. http://informesdelaconstruccion.revistas. csic.es/index.php/informesdelaconstruccion/article/viewArticle/4581

Heschong, L. (1999). Daylighting in Schools: An Investigation into the Relationship Between Daylighting and Human Performance. Submitted by the Heschong Mahone Group to Pacific Gas and Electric, on behalf of the California Board for Energy Efficiency Third Party Program. http://h-m-g.com/downloads/Daylighting/ schoolc.pdf

Heschong, L., Roger, L., \& Okura, S. (2002). Daylighting Impacts on Human Performance in School, Journal of the Illuminating Engineering Society (Summer), 101 114. http://c.ymcdn.com/sites/www.co-case.org/resource/resmgr/imported/ JA Daylighting $\% 20$ Impacts $\% 20$ on $\% 20$ Human $\% 20$ Performance $\% 20$ in $\% 20$ Schools.pdf

Holmberg, K., Landstrom, U., \& Kjellberg, A. (1993). Effects of ventilation noise due to frequency characteristic and sound level. Journal of Low Frequency Noise and Vibration, 4, 115-122. http://lfn.sagepub.com/content/12/4/115.refs

Ison, M. S., \& Pattini, A. E. (2009). Eficacia Atencional en Niños y Optimización del Acondicionamiento Lumínico en Aulas. Investigación en Ciencias del Comportamiento. Avances Iberoamericanos. Buenos Aires: CIIPME- CONICET. http://www.cricyt. edu.ar/asades/modulos/averma/trabajos/2010/2010-t001-a008.pdf

Ison, M. S. (2011). Programa de intervención para mejorar las capacidades atencionales en escolares argentinos. International Journal of Psychological Research, 4(2), 72-79. http://www.redalyc.org/html/2990/299023516009/

Ison, M. S., \& Korzeniowski, C. (2016). El Rol de la Atención y Percepción Viso-Espacial en el Desempeño Lector en la Mediana Infancia. Psykhe, 25(1), 1-13. doi:10.7764l psykhe.25.1.761

Ison, M. S., Greco, C., Korzeniowski, C., \& Morelato, G. (2015). Attentional Efficiency: A Comparative Study on Argentine Students Attending Schools from Different Socio-Cultural Contexts. Electronic Journal of Research in Educational Psychology, 13(2), 343-368. doi:10.14201/ejrep.36.14092

Ison, M. S., Korzeniowski, C., Segretin, M., \& Lipina, S. (2015). Evaluación de la eficacia atencional en niños argentinos sin y con extraedad escolar. Revista Argentina de Ciencias del Comportamiento, 7(1), 38-52. www.redalyc.org/ pdf/3334/333439929005.pdf

Ison, M. S., \& Carrada, M. (2011). Evaluación de la eficacia atencional: Estudio normativo preliminar en escolares argentinos [Assessment of attentional efficiency: Preliminary normative study in Argentine school]. RIDEP, 29, 129-146. http:// alfama.sim.ucm.es/wwwisis2/wwwisis.exe/[in=psyke2.in]/?format=breve\&boo lean $=\% 5 B N R: 11353848$

Knez, I. (1997). Changes in females and males' positive and negative moods as a result of variations in CCT, CRI and illuminance levels. En Proceedings of Right Light: 4th European Conference on Energy Eficient Lighting, Copehagen Denmark, Vol. 1, 149-154. http://eab.sagepub.com/content/30/4/553.abstract

Kuller, R., \& Lindsten, C. (1992). Health and Behavior of Children in Classrooms with and without Windows. Journal of Environmental Psychology, (12), 305-317. https://www.researchgate.net/publication/248587968 Health and behavior of children in classrooms with and without windows

Lesesne, C. A., Visser, S. N., \& White, C. P. (2003). Attention-Deficit / Hyperactivity Disorder in school-aged children: Association with Maternal Mental Health and Use of Health Care Resources. Pediatrics, 111, 1232-1237. Google books 
Matute, E., Sanz, A., Gumá, E., Roselli, M., \& Ardila, A. (2009). Influencia del nivel educativo de los padres, el tipo de escuela y el sexo en el desarrollo de la atención y la memoria. Revista Latinoamericana de Psicología, 41(2), 257-273. doi:1434/rlp. $\underline{\mathrm{v} 41 \mathrm{i} 2.380}$

Mezzacappa, E. (2004). Alerting, Orienting, and Executive Attention: Developmental Properties and Sociodemographic Correlates in an Epidemiological Sample of Young, Urban Children. Child Development, 75(5), 1373-1386. http://onlinelibrary. wiley.com/doi/10.1111/j.1467-8624.2004.00746.x/abstract

Mills, P. R., Tomkins, S. C., \& Schlangen, L. J. (2007). The effect of high correlated colour temperature office lighting on employee wellbeing and work performance. Journal of Circadian Rhythms, 5(1), 2. doi: http://dx.doi.org/10.1186/1740-3391$\underline{5-2 .}$

Monteoliva, J. M. (2014). Iluminación en aulas. La dinámica de luz natural en cielos claros y su incidencia en el rendimiento atencional de los alumnos. (Tesis Doctoral inédita). Facultad de Ciencias Exactas y Tecnología, Universidad Nacional de Tucumán, San Miguel de Tucumán, Argentina.

Monteoliva, J. M., Ison, M. S., \& Pattini, A.E. (2014). Evaluación del desempeño atencional en niños: Eficacia, eficiencia y rendimiento. Interdisciplinaria, 31(2), 213-225. http://www.redalyc.org/pdf/180/18032537002.pdf

Okura, S., Heschong L., \& Wright, R. (2000). Skylighting and Retail Sales. ACEEE Summer Study on Energy Efficiency in Buildings, 8, 245-56.

Pattini, A. (2009). La Luz Natural en las Escuelas: aprovechamiento y control de la luz solar en aulas. Buenos Aires: Dunken. http://www.scielo.br/scielo.php?script=sci arttext\&pid=S1678-86212014000300004

Polderman, T., Boomsma, D., Bartels, M., Verhulst, F., \& Huizink, A. (2010). A systematic review of prospective studies on attention problems and academic achievement. Act Psychiatr Scand, 122, 271-284. http://www.ncbi.nlm.nih.gov/pubmed/20491715

Posner, M. I., \& Rothbart, M. K. (2014). Attention to learning of school subjects. Trends in Neuroscience and Education, 3, 14-17. doi:10.1016/j.tine.2014.02.003

Posner, M., Rothbart, M., \& Rueda, M. (2011). Desarrollo de la autorregulación y desempeño escolar. En Lipina, S. J. \& Sigman, M. (Eds), La pizarra de Babel. Puentes entre neurociencia, psicología y educación (pp.133-152). Buenos Aires: Libros Del Zorzal. http://grados.ugr.es/psicologia/pages/infoacademica/tfgspropuestos $1617 / !$

Rea, M. S. (2002). Light, Much More Than Vision. En Proceeding of Light and Human Health: EPRI/LRO 5th International Lighting Research Symposium, 1-15. Palo Alto, CA, USA: The Lighting Research Office of the Electric Power Research Institute,

Ridderinkhof, K. R., \& Van Der Molen, M. W. (1995). A psychophysiological analysis of developmental differences in the ability to resist interference. Child Development, 66, 1040-1056. Google books

Rosselli, M., \& Ardila, A. (2003). The impact of culture and education on non verbal neuropsychological measures: A critical review. Brain and Cognition, 52, 326-33. http://www.ncbi.nlm.nih.gov/pubmed/12907177

Sanaz, A. S. (2011). The Influence of Light on Student's Learning Performance in Learning Environments: A Knowledge Internalization Perspective. Journal of World Academy of Science, Engineering and Technology, 81. http://www.sciepub. com/reference/12931

Sheese, B., Rothbart, M., Posner, M., White, L., \& Fraundorf, S. (2008). Executive attention and self-regulation in infancy. Infant Behavior and Development, 31, 501 510. https://www.researchgate.net/publication/222552260 Executive attention and self-regulation in infancy 
Thurstone, L. L., \& Yela, M. (2012). CARAS-R - Percepción de diferencias [FACES - Perception of differences]. Madrid: TEA Ediciones. http://www.web.teaediciones. com/Ejemplos/CARAS-R\%20Manual 2012.pdf

Tonello, G. (1998). Efectos no visuales de la luz, una puesta al día. Revista Luminotecnia, 56, 1-8.

Van Bommel, W.J.M., \& Van Den Beld, G.J. (2004). Lighting for work: a review of visual and biological effects. Lighting Research \& Technology, 36(4), 255-269. doi: http://dx.doi.org/10.1191/1365782804li122oa.

Veitch, J. A., \& Newsham, G. R. (1998). Determinants of lighting quality 1: State of science. Journal of the Illuminating Engineering Society, 27(1), 92-106. http://www. tandfonline.com/doi/abs/10.1080/00994480.1998.10748215

Veitch, J. A. (1997). Revisiting the performance and mood effects of information about lighting and fluorescent lamp type. Journal of Env. Psychology, 17, 253262. http://citeseerx.ist.psu.edu/viewdoc/download?doi=10.1.1.2.7066\&rep=re p1\&type $=$ pdf

Veitch, J. A., \& Newsham, G. R. (1998). Lighting quality and energy-efficiency effects on task performance, mood, health, satisfaction and comfort. Journal of IndoEuropean Studies, 27, 107-129.

Waechter, S., Besner, D., \& Stolz, J. (2011). Basic processes in reading: Spatial attention as a necessary preliminary to orthographic and semantic processing. Visual cognition, 19(2), 171-202. doi:10.1080/13506285.2010.517228

Webb, A. R. (2006). Considerations for lighting in the built environment: Non-visual effects of light. Energy and Buildings, 38, 721-727. http://Irt.sagepub.com/content/44/1/37.abstract

Welsh, J. A., Nix, R. L., Blair, C., Bierman, L., \& Nelson, K. E. (2010). The Development of Cognitive Skills and Gains in Academic School Readiness for Children from LowIncome Families. Journal of Educational Psychology, 102(1), 43-53. doi: 10.1037/ $\underline{\mathrm{a} 0016738}$

Wesnes, K. A., Pincock, C., Richardson, D., Helm, G., \& Hails, S. (2003). Breakfast reduces declines in attention and memory over the morning in school children. Appetite, 41, 329-331. doi:10.1016/j.appet.2003.08.009

Wienold, J., \& Christoffersen, J. (2006). Evaluation methods and development of a new glare prediction model for daylight environments with the use of CCD cameras. Energy and Buildings, 38(7), 743-757. https://www.researchgate.net/publication/222908061 Evaluation methods and development of a new glare prediction model for daylight environments with the use of CCD cameras

Wu, W., \& Ng, E. (2003). A review of the development of daylighting in schools. Lighting Research and Technology, 35, 111-125. http://lrt.sagepub.com/content/35/2/111.refs 